\title{
Research on Adaptive Scheduling Algorithm Based on Improved Genetic Algorithm for Multifunctional Phased Array Radar
}

\author{
Shuaijie Wang, Jun He, Bin Wang, Ruilong Ji \\ Department of operational research, University of Electronic Engineering Institute Hefei, Anhui Province, China \\ wsj3004@126.com
}

\begin{abstract}
Because of agile beam capability, Multifunctional phased array radar (MFPAR)can perform not only search and track targets but also test and verify targets. Therefore an effective scheduling tactics is required so that the time and energy resources can be effectively allocated to optimize the overall performance of MFPAR. Analyzing the design principle of adaptive scheduling algorithm for MFPAR, this paper presents specific indexes to evaluate performance. Aiming at MFPAR, this paper uses improved genetic algorithm as adaptive scheduling algorithm. The algorithm can reasonably schedule radar tasks in the scheduling interval on the basis of their priority, dwelling length, comprehensive efficiency and the time constraint of scheduling interval. The validity of scheduling algorithm is proved by the results of simulation.
\end{abstract}

Index Terms - phased array radar, genetic algorithm, scheduling interval, comprehensive efficiency

\section{Introduction}

The performance of the phased array radar scheduling strategy is crucial to taking advantage of its flexible beam scanning [1]. The design method of the phased array radar scheduling strategy has many ways, among which fixed template, multi-templates, part template and self-adaptive scheduling algorithm are usually used[2].Currently the self-adaptive scheduling algorithm is the most effective design method for phased array radar. Reference [2] has given a definition of the self-adaptive scheduling strategy. Based on the work above, this paper puts forward a improved genetic self-adaptive strategy, which aims at maximizing the efficiency of phased array radar in limited time. The simulation results shows the scheduling algorithm from this paper can improve the scheduling efficiency and time utilization of the phased array radar in limited radar time resources.

\section{The Design of Scheduling Strategy and Performance Evaluation Indexes}

The overall structure of the phased array radar scheduling strategy is shown in Fig.1, and the main task of the radar is shown in TABLE 1 . The computer system of radar summarizes all the radar tasks, analyzes them preliminarily, evaluates the threat degree for each task in some way, and further divides these tasks into different priority levels. Puts the task with priority level into a scheduler, schedules the resources in the self-adaptive scheduling algorithm from this paper in limited time, and gets three schedule lists(task execution list, task delay list and task abandon list).The task execution list refers to the radar tasks which need to be executed immediately at current scheduling interval, and radar antenna system displays rational beams according to the task parameter. The task delay list refers to the not so important tasks which cannot be executed immediately for current radar resource limit, and they are reput to the 1 radar task overall task at next schedule interval to schedule twice. Task abandon list is used to store unimportant radar task in order to reduce unnecessary radar resource needs. For example, a radar task is to trace a certain target, its threat degree don't need to trace precisely, which only a small amount of tracing beams will do. This task's comprehensive effectiveness is low according to the self-adaptive scheduling algorithm, thus it will be put in the task abandon list when other tasks are busy. Therefore, radar tasks can be ranked adaptively according to each effectiveness value using this scheduling model, enabling the rational distribution of radar resources in continuously variable circumstances.

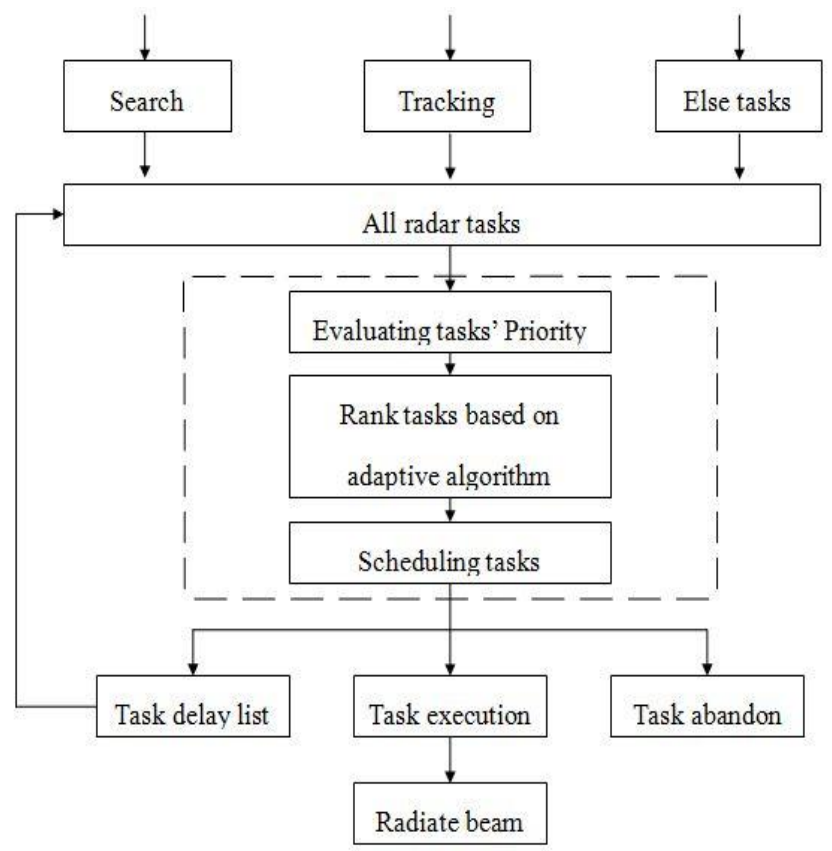

Fig.1 The overall structure of the phased array radar scheduling strategy 
TABLE 1 The main task of MFPAR

\begin{tabular}{|c|c|c|c|}
\hline Identifier & The type of radar task & Priority & Dwell time \\
\hline 1 & Verification & 6 & $6 \mathrm{~ms}$ \\
\hline 2 & Precision tracking & 5 & $2 \mathrm{~ms}$ \\
\hline 3 & High priority search & 4 & $6 \mathrm{~ms}$ \\
\hline 4 & Common tracking & 3 & $4 \mathrm{~ms}$ \\
\hline 5 & Missing processing & 2 & $5 \mathrm{~ms}$ \\
\hline 6 & Low priority search & 1 & $5 \mathrm{~ms}$ \\
\hline
\end{tabular}

\section{A. Principle of the scheduling algorithm}

\section{1) Priority principle:}

Make the length of scheduling interval $\mathrm{T}$, the set of tasks during the interval $\mathrm{R}=\left\{\mathrm{r}_{1}, \mathrm{r}_{2}, \ldots, \mathrm{r}_{n}\right\} . \forall \mathrm{r}_{i}, \mathrm{r}_{j}$ when $\mathrm{r}_{i}$ and $\mathrm{r}_{j}$ compete for the same time slot, if $\mathrm{r}_{i}$ priority is $\mathrm{Y}_{i}, \mathrm{r}_{j}$ priority is $\mathrm{Y}_{j}$, then $\mathrm{Y}_{i}>\mathrm{Y}_{j}, \mathrm{r}_{i}$ is scheduled preferentially, when $\mathrm{Y}_{i}<\mathrm{Y}_{j}$, and vice versa.

\section{2) Time utilization principle}

Make the task $\mathrm{r}_{i}$ persistence length of scheduling interval $\Delta \mathrm{T}_{i}$, then:

$$
\text { s.t }\left\{\begin{array}{l}
\sum_{i=1}^{n} \Delta \mathrm{T}_{i} \leq \mathrm{T} \\
\sum_{i=1}^{n} \Delta \mathrm{T}_{i} \rightarrow \mathrm{T}
\end{array}\right.
$$

\section{3) efficiency maximization principle :}

At the same scheduling interval, radar tasks cannot maximize the comprehensive effectiveness of phased array radar only according the priority ranking, the beneficial impact factor of the same priority ranking tasks at different time ranking should be taken into consideration. If the priority of losing trace is 2 (TABLE 1), but it ranks first at the same scheduling interval, then its beneficial impact factor is 0.9 according to the actual situation and expert experience. If it ranks last at the same scheduling interval, then its beneficial impact factor is 0.1 because the phased array radar may lose its trace permanently as time goes by. The scheduling algorithm should rank each radar task according to each task's priority, persistence length and beneficial impact factor, thus maximizing comprehensive effectiveness.

\section{B. Performance evaluation indexes and self-adaptive scheduling model}

To evaluate the comprehensive effectiveness of phased array radar at the same scheduling interval, this paper defines radar task capacity and beneficial impact factor, and establishes self-adaptive scheduling model based on the scheduling principle above.

1) Single radar task capacity $\left(R E V_{i}\right)$

$$
R E V_{i}=g\left(\mathrm{Y}_{i}, \Delta \mathrm{T}_{i}\right)
$$

Among this: $g(\bullet)$ is capacity function, designed comprehensively according to priority principle and time utilization principle. $R E V_{i}$ is larger if priority higher and persistence length longer, and vice versa. The design of $g(\bullet)$ has a certain amount of flexibility, adopting the forms below :

$$
g\left(\mathrm{Y}_{i}, \Delta \mathrm{T}_{i}\right)=\left(1+a * \mathrm{Y}_{i}\right) * \Delta \mathrm{T}_{i}^{b}
$$

Among this: $a$ and $b$ are adjustment coefficient $(a, b \geq 0, a+b=1$ ), the larger $a$ gets, the smaller $b$ gets. The more $\mathrm{Y}_{i}$ influence $R E V_{i}$, the less $\Delta \mathrm{T}_{i}$ does. When $a$ equals 0 , then $\mathrm{Y}_{i}$ has no influence towards $R E V_{i}$, and when $b$ equals 0 , then $\Delta \mathrm{T}_{i}$ has no influence towards $R E V_{i}$.

\section{2) Beneficial impact factor $U_{i j}$}

$U_{i j}$ is the beneficial impact factor which ranks No. $i$ at scheduling interval $\mathrm{T}$, and mainly determined according to the actual situation and expert experience. If the scheduler needs to deal with 9 radar tasks at certain scheduling interval, then the beneficial impact matrix is

$$
U=\left(\begin{array}{ccccc}
U_{11} & U_{12} & \cdots & \cdots & U_{19} \\
U_{21} & \ddots & & & \vdots \\
\vdots & & \ddots & & \vdots \\
\vdots & & & \ddots & U_{89} \\
U_{91} & \cdots & \cdots & U_{98} & U_{99}
\end{array}\right)
$$

\section{3) Self-adaptive scheduling model}

Contraposing the performance evaluation indexes, the self-adaptive scheduling model is established like what is shown below:

$$
\begin{aligned}
& E_{\max }=\max \left\{\sum_{j=1}^{n} g\left(\mathrm{Y}_{i}, \Delta \mathrm{T}_{i}\right) * U_{i j}\right\} \\
& \text { s.t }\left\{\begin{array}{l}
\sum_{i=1}^{n} \Delta \mathrm{T}_{i} \leq \mathrm{T} \\
\sum_{i=1}^{n} \Delta \mathrm{T}_{i} \rightarrow \mathrm{T}
\end{array}\right.
\end{aligned}
$$

The comprehensive effectiveness $E_{\max }$ of phased array radar is calculated by the self-adaptive scheduling algorithm, then identify the radar task which should be put in the task execution list at scheduling interval $\mathrm{T}$, put the radar tasks which are outside the scheduling interval into task delay list and task abandon list according to certain judgement standards[5]. Fig.1 shows the specific process. 


\section{The Improved Genetic Algorithm for Scheduling of Phased Array Radar}

\section{A. Traditional genetic algorithm steps}

The Fig.2 shows the program flow of the traditional genetic algorithm [3].

\section{B. The improved genetic algorithm for scheduling of phased array radar}

Phased array radar scheduling interval time in millisecond, determines its search for a short time. Additionally, we need to find the global optimal solution as much as possible, and scheduling interval maximum comprehensive efficiency. This is a unity of opposites of the problems existing in traditional genetic algorithm: the search efficiency and the search space of the problem, namely the problem of direction and randomness. Enhancing the directivity can improve the efficiency of genetic algorithm, increasing the randomness can increase the global searching of genetic algorithm, these two factors determine the performance of genetic algorithm: these two aspects of equilibrium are directly determined by the crossover probability $\mathrm{Pc}$ and mutation probability $\mathrm{Pm}$.

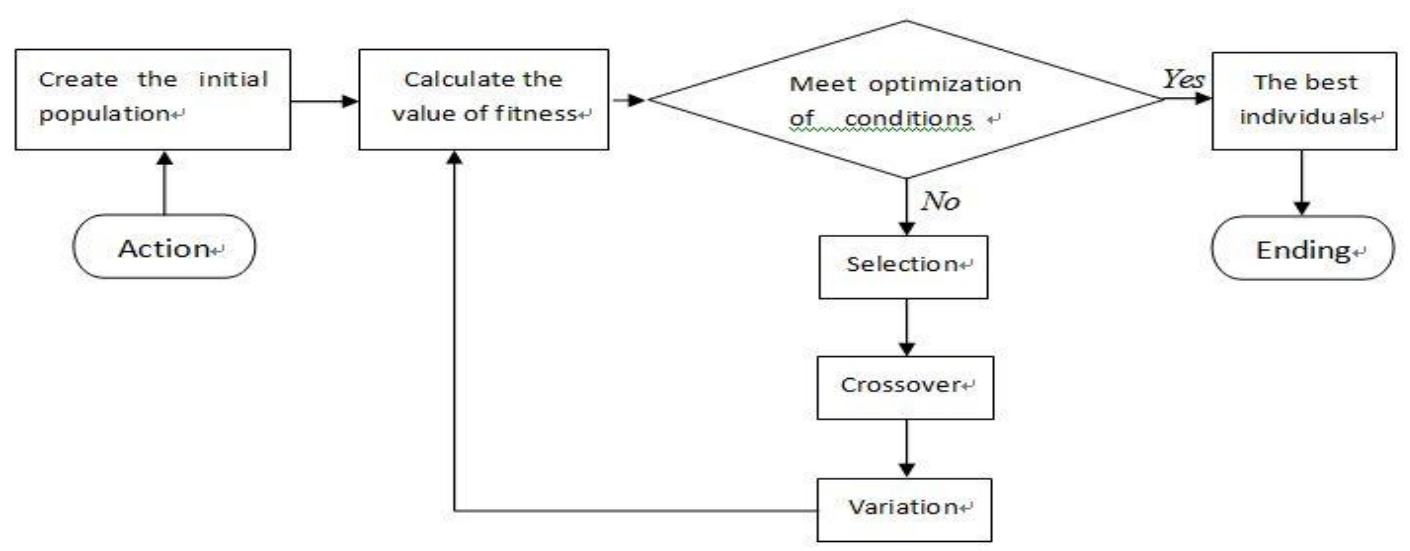

Fig.2 The program flow of the traditional genetic algorithm

The crossover probability controls probability of crossover operation being used. The larger crossover probability can enhance the ability of genetic algorithm to open the search area, but the possibility of high performance mode being damaged also increases. If crossover probability is too low, the genetic algorithm may fall into a torpid state. Mutation operation belongs to the auxiliary operation in the genetic algorithm, and its purpose is to keep the diversity of the swarm. Generally, variation of low frequency can prevent the loss of an important, single gene in a population. Variation of high frequency will make genetic algorithm tends to pure random search.

In order to make the genetic algorithm own higher global optimality and efficiency, we increase the crossover probability $\mathrm{Pc}$ and mutation probability $\mathrm{Pm}$ when the algorithm is convergent in advance ideally. Reduce $\mathrm{Pc}$ and Pm early in the algorithm, so as not to damage the searching efficiency of genetic algorithm. For the high fitness individuals, we use low $\mathrm{Pc}$ and $\mathrm{Pm}$, in order to maintain the excellent individuals. For the lower fitness individuals, we use higher $\mathrm{Pc}$ and $\mathrm{Pm}$, in order to promote the evolution of the individual. The size of $\mathrm{Pc}$ and $\mathrm{Pm}_{\text {is not only connected with }}$ convergence of the algorithm, but also related with individual fitness. This paper introduces the improved adaptive formula[4], which is improved from adaptive crossover rate and mutation rate proposed by Striniras. As the following shows us:

$$
\begin{aligned}
& P c= \begin{cases}\text { Pc1- } \frac{(P c 1-P c 2)\left(f^{\prime}-f_{a v g}\right)}{f_{\text {max }}-f_{\text {avg }}} & f^{\prime} \geq f_{\text {avg }} \\
\text { Pc1 } & f^{\prime}<f_{\text {avg }}\end{cases} \\
& P m= \begin{cases}P m 1-\frac{(P m 1-P m 2)\left(f_{\text {max }}-f\right)}{f_{\text {max }}-f_{\text {avg }}} & f^{\prime} \geq f_{\text {avg }} f_{\text {max }}: \\
\text { Pm1 } & f^{\prime}<f_{\text {avg }}\end{cases}
\end{aligned}
$$

The maximum fitness value of the population

$f_{\text {avg }}$ : The average fitness value of each generation group

$\mathrm{f}^{\prime}$ : The larger fitness value in two crossover individuals

$\mathrm{f}$ : The fitness of variation individuals

$$
\text { Generally, } \mathrm{Pcl}=0.9, \mathrm{Pc} 2=0.6, \mathrm{Pml}=0.1 \text {, }
$$

$\mathrm{Pm} 2=0.01$.According to the actual situation, the numbers can be adjusted properly. From the above formulas, we can see that, $\mathrm{f}_{\text {max }}-\mathrm{f}_{\text {avg }}$ Is a measure of the algorithm's convergence degree. When the algorithm converges to a local optimal value, $f_{\text {max }}-f_{\text {avg }}$ become lower. At this point, we should increase 
$\mathrm{Pc}_{\text {and }} \mathrm{Pm}$, in order to make the algorithm escape from local optima as soon as possible. ${ }^{\prime}-f_{\text {avg }}$ or $f_{\text {max }}-f_{\text {avg }}$ is used to measure the individual's excellent degree. When the fitness value is lower than the average fitness value, corresponding $f^{\prime}-f_{\text {avg }}$ or $f_{\text {max }}-f$ will be increased. It tells that the performance of this individual is not good. And we should use the larger $\mathrm{Pc}$ and $\mathrm{Pm}$; the $\mathrm{f}^{\prime}-\mathrm{f}_{\text {avg }}$ or $\mathrm{f}_{\max }-\mathrm{f}$ of the individuals which own high value of fitness reduces. At this point, $\mathrm{Pc}_{\text {and }} \mathrm{Pm}$ will be decreased to ensure the excellent individuals not damaged or missing for crossover and mutation.

\section{Case Analysis}

Assuming the scheduling interval is $50 \mathrm{~ms}, \mathrm{~T}=50 \mathrm{~ms}$.There are 12 radar tasks, whose

task sets $\quad$ is: $R=\left\{\begin{array}{lll}r_{1}, & r_{2} \ldots, & r_{12}\end{array}\right\}$

TABLE $2 R E V$ of tasks

\begin{tabular}{|c|c|c|c|c|c|c|c|c|c|c|c|c|}
\hline The task number & $\mathrm{r}_{1}$ & $\mathrm{r}_{2}$ & $\mathrm{r}_{3}$ & $\mathrm{r}_{4}$ & $\mathrm{r}_{5}$ & $\mathrm{r}_{6}$ & $\mathrm{r}_{7}$ & $\mathrm{r}_{8}$ & $\mathrm{r}_{9}$ & $\mathrm{r}_{10}$ & $\mathrm{r}_{11}$ & $\mathrm{r}_{12}$ \\
\hline Identifier & 1 & 4 & 2 & 6 & 3 & 5 & 1 & 2 & 3 & 6 & 4 & 4 \\
\hline Priority & 6 & 3 & 5 & 1 & 4 & 2 & 6 & 5 & 4 & 1 & 3 & 3 \\
\hline Dwell time & $6 \mathrm{~ms}$ & $4 \mathrm{~ms}$ & $2 \mathrm{~ms}$ & $5 \mathrm{~ms}$ & $6 \mathrm{~ms}$ & $5 \mathrm{~ms}$ & $6 \mathrm{~ms}$ & $2 \mathrm{~ms}$ & $6 \mathrm{~ms}$ & $5 \mathrm{~ms}$ & $4 \mathrm{~ms}$ & $4 \mathrm{~ms}$ \\
\hline$R E V$ & 9.798 & 5 & 4.9497 & 3.3541 & 7.3485 & 4.4721 & 9.798 & 4.9497 & 7.3485 & 3.3541 & 5 & 5 \\
\hline
\end{tabular}

Their task properties can be seen from TABLE 1. when $a=0.5$ and $b=0.5, R E V$ of each task can be seen from TABLE 2.

$U=\left[\begin{array}{llllll}0.91 & 0.52 & 0.82 & 0.55 & 0.85 & 0.95 \\ 0.81 & 0.42 & 0.72 & 0.45 & 0.75 & 0.85 \\ 0.72 & 0.33 & 0.63 & 0.36 & 0.66 & 0.76 \\ 0.64 & 0.25 & 0.55 & 0.28 & 0.58 & 0.68 \\ 0.60 & 0.21 & 0.51 & 0.24 & 0.54 & 0.64 \\ 0.57 & 0.18 & 0.48 & 0.21 & 0.51 & 0.61 \\ 0.55 & 0.46 & 0.46 & 0.19 & 0.49 & 0.59 \\ 0.54 & 0.36 & 0.36 & 0.55 & 0.48 & 0.58 \\ 0.53 & 0.26 & 0.26 & 0.45 & 0.47 & 0.57 \\ 0.52 & 0.16 & 0.25 & 0.36 & 0.46 & 0.56 \\ 0.51 & 0.15 & 0.24 & 0.35 & 0.45 & 0.55 \\ 0.50 & 0.14 & 0.23 & 0.34 & 0.44 & 0.54\end{array}\right.$

Assuming the time and resource is constraint, make comprehensive efficiency $\mathrm{E}$ trend to $\mathrm{E}_{\max }$.We use the improved genetic algorithm to solve this problem. Parameter settings is that, population $\mathrm{NC}$ is 30,the largest genetic algebra GEN is 50 , the length of chromosome $\mathrm{m}$ is 12 ,
According to the actual situation and the experience of experts, we get the beneficial matrix $U$ through a certain algorithm [6].

$\left.\begin{array}{llllll}0.91 & 0.82 & 0.85 & 0.55 & 0.52 & 0.52 \\ 0.81 & 0.72 & 0.75 & 0.45 & 0.42 & 0.42 \\ 0.72 & 0.63 & 0.66 & 0.36 & 0.33 & 0.33 \\ 0.64 & 0.55 & 0.58 & 0.28 & 0.25 & 0.25 \\ 0.60 & 0.51 & 0.54 & 0.24 & 0.21 & 0.21 \\ 0.57 & 0.48 & 0.51 & 0.21 & 0.18 & 0.18 \\ 0.55 & 0.46 & 0.49 & 0.19 & 0.46 & 0.46 \\ 0.54 & 0.36 & 0.48 & 0.55 & 0.36 & 0.36 \\ 0.53 & 0.26 & 0.47 & 0.45 & 0.26 & 0.26 \\ 0.52 & 0.25 & 0.46 & 0.36 & 0.16 & 0.16 \\ 0.51 & 0.24 & 0.45 & 0.35 & 0.15 & 0.15 \\ 0.50 & 0.23 & 0.44 & 0.34 & 0.14 & 0.14\end{array}\right]$

crossover probability $\mathrm{Pc} 1$ is $0.9, \mathrm{Pc} 2$ is 0.6 , and the mutation probability $\mathrm{Pm} 1$ is $0.1, \mathrm{Pm} 2$ is 0.01 .

Solve the problem with Matlab. After 50 iterations, the target assignment result is shown in TABLE 3 .

TABLE 3 The allocation results

\begin{tabular}{|c|c|c|c|c|c|c|c|c|c|c|c|c|}
\hline The task number & $\mathrm{r}_{1}$ & $\mathrm{r}_{2}$ & $\mathrm{r}_{3}$ & $\mathrm{r}_{4}$ & $\mathrm{r}_{5}$ & $\mathrm{r}_{6}$ & $\mathrm{r}_{7}$ & $\mathrm{r}_{8}$ & $\mathrm{r}_{9}$ & $\mathrm{r}_{10}$ & $\mathrm{r}_{11}$ & $\mathrm{r}_{12}$ \\
\hline The allocation results & 2 & 7 & 10 & 8 & 4 & 5 & 1 & 6 & 3 & 9 & -12 & 11 \\
\hline
\end{tabular}

The total efficiency of this scheme is 39.5442 .

Fig. 3 shows us the objective function of optimization solution after 50 iterations. From the simulation results, we can see that the optimal solution can be gotten after 22 iterations. 


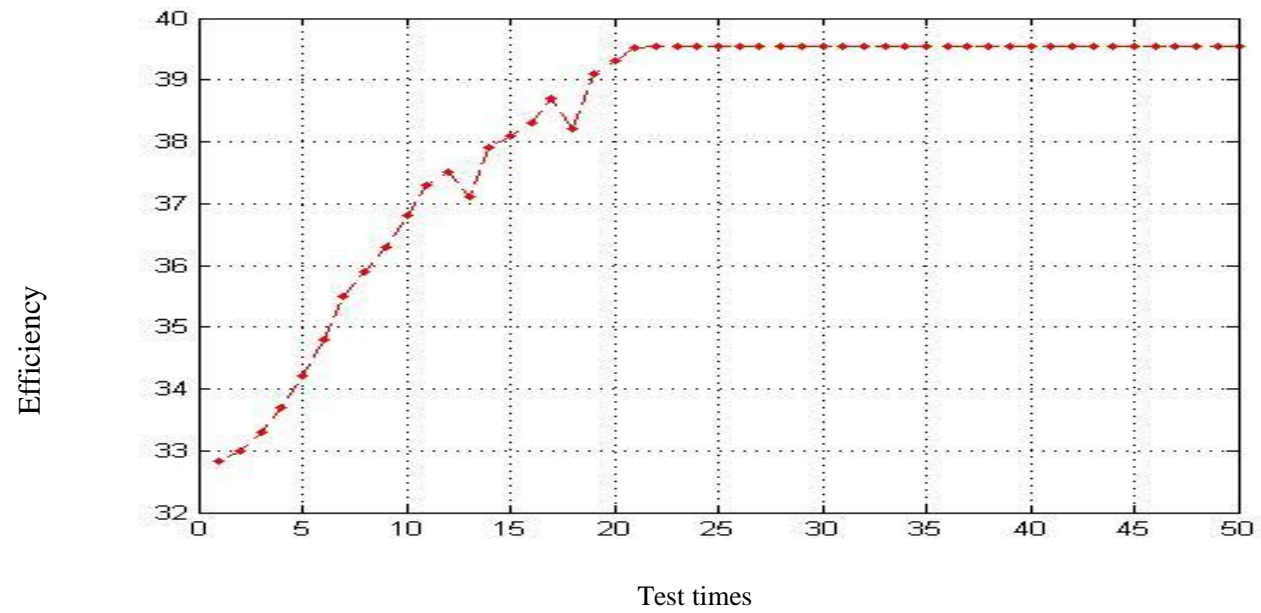

Fig.3 The simulation results

According to time constraint formula (2), when the scheduling interval is $50 \mathrm{~ms}$, we can arrange task $r_{7}, r_{1}, r_{9}, r_{5}, r_{6}, r_{8}, r_{2}, r_{4}, r_{10}, r_{3}$, the time totally is $47 \mathrm{~ms}$. Task $\mathrm{r}_{11}, \mathrm{r}_{12}$ will be put into delay task list, abandon the task list through certain standards of judgment[6].

\section{Conclusions}

The paper improves the probability of crossover and mutation to make genetic algorithm better. It not only enhances efficiency of GA but also increases the probability of global optimum. The paper's simulation is on the basis of adaptive scheduling algorithm. It presents detailed process of scheduling algorithm, quantitatively analyses scheduling efficiency. The simulation results indicates that scheduling algorithm with time constraint of scheduling interval can make comprehensive efficiency maximum - it increases time use ratio of MFPAR on the basis of priority principle.

The scheduling tactics of phased array radar should consider the constraints of time, energy and computer resource.

The paper only considers the constraint of time, and should further research adaptive scheduling algorithm on the comprehensive constraints of time, energy and computer resource.

\section{References}

[1] Guangyi Zhang. The System of Phased Array Radar. Beijing: National Defence Industrial Press, 1994.

[2] Liancheng Wang. Computer controls Modern Radar. Beijing: Aerospace Industry Department, 1973.

[3] Dethloff J.Vehicle, "Routing and Reverse Logistics: the Vehicle Routing Problem with Simultaneous Delivery and Pick-up," OR Spektrum, in press.

[4] Min Liu, et a1, "An Adaptively Annealing Genetic Algorithm based Scheduling Method of Workshop Daily Operating Planning," Chinese Journal of Computers, pp.1164-1172, July 2007.

[5] Guang Zeng, "Research on Adaptive Scheduling Algorithm for Multifunction Phased Array Radar," Modern Radar, in press.

[6] Shiyou Zheng. "Adaptive Task Scheduling Algorithm Based on Synthesizing Programming for Phased Array Radar," Computer Simulation, in press.

- Shuai-jie Wang (1989-), Postgraduate, School of Electronic Engineering Institute, HeFei of China,13866102474,wsj3004@126.com., His research interest is efficiency estimation.

- Jun He: Ph.D.adviser, School of Electronic Engineering Institute, HeFei of China. His research interest is efficiency estimation.

- Bin Wang: Supervisor of postgraduate, School of Electronic Engineering Institute. HeFei of China. His research interest is efficiency estimation.

- Rui-long Ji: Postgraduate, School of Electronic Engineering Institute, hefei. His research interest is efficiency estimation. 